\title{
Protective effects of different anti-inflammatory drugs on tracheal stenosis following injury and potential mechanisms
}

\author{
ZHENJIE HUANG ${ }^{1}$, PENG WEI $^{1}$, LUOMAN GAN $^{2}$, WENTAO LI $^{1}$, TONGHUA ZENG ${ }^{3}$, \\ CAICHENG QIN $^{1}$, ZHIYU CHEN $^{3}$ and GUANGNAN LIU ${ }^{1}$ \\ ${ }^{1}$ Department of Respiratory Medicine, Second Affiliated Hospital of Guangxi Medical University, \\ Nanning, Guangxi 530007; ${ }^{2}$ School of Medicine, Qinghai University, Xining, Qinghai 810000; \\ ${ }^{3}$ Department of Respiratory Medicine, Beihai People's Hospital, Beihai, Guangxi 536000, P.R. China
}

Received November 28, 2019; Accepted July 7, 2020

DOI: $10.3892 / \mathrm{mmr} .2021 .11953$

\begin{abstract}
Tracheal stenosis following injury cannot be effectively treated. The current study compared the protective effects of different anti-inflammatory drugs on tracheal stenosis and investigated their possible mechanisms. Rabbit tracheal stenosis models following injury were constructed and confirmed using hematoxylin and eosin (H\&E) staining. A total of 30 rabbits were divided into the control (CON), penicillin (PEN), erythromycin (ERY), budesonide (BUD) and PEN + ERY + BUD groups $(n=6)$. Stenotic tracheal tissue, serum and bronchoalveolar lavage fluid (BALF) were collected 10 days after continuous treatment. Pathological changes in the tracheas were observed by H\&E staining. Histone deacetylase 2 (HDAC2) expression in tracheal tissues was detected by immunofluorescence. Immunohistochemistry was performed to detect collagen I (Col-I) and collagen III (Col-III) levels in tracheal tissues. Transforming growth factor $\beta 1$ (TGF- $\beta 1$ ), vascular endothelial growth factor (VEGF) and interleukin 8 (IL-8) levels in serum and BALF samples were determined using ELISA kits. Western blotting detected HDAC2, IL-8, TGF- $\beta 1$ and VEGF levels in tracheal tissues. H\&E staining demonstrated that tracheal epithelial hyperplasia and fibroblast proliferation in the ERY and PEN + ERY + BUD groups markedly improved compared with the CON group. Furthermore, in tracheal tissues, HDAC2 expression was significantly increased and IL-8, TGF- $\beta 1$, VEGF, Col-I and Col-III levels were significantly decreased in the ERY and PEN + ERY + BUD groups compared with the CON group. Additionally, the results for the PEN + ERY + BUD were more significant compared with the ERY group. In serum and BALF samples, IL-8, TGF- $\beta 1$ and
\end{abstract}

Correspondence to: Dr Guangnan Liu, Department of Respiratory Medicine, Second Affiliated Hospital of Guangxi Medical University, 166 Daxuedong Road, Nanning, Guangxi 530007, P.R. China

E-mail: gnliu63@hotmail.com

Key words: anti-inflammatory drugs, tracheal stenosis, histone deacetylase 2, granulation tissue
VEGF levels in the ERY and PEN + ERY + BUD groups were significantly lower compared with the CON group, with the results of the PEN + ERY + BUD group being more significant compared with the ERY group. There were no significant differences between the PEN, BUD and CON groups. ERY inhibited tracheal granulation tissue proliferation and improved tracheal stenosis following injury and synergistic effects with PEN and BUD further enhanced these protective effects. The mechanism may involve HDAC2 upregulation and inhibition of local airway and systemic inflammatory responses.

\section{Introduction}

With the increasing requirement for severe medical and respiratory support, the incidence of tracheal stenosis caused by tracheal intubation or tracheotomy is gradually increasing in China over the past ten years (1). Although surgical and endoscopic interventions have several effects on benign tracheal stenosis, secondary injury and excessive healing following trauma can easily lead to tracheal restenosis (2). Therefore, drug treatments for the healing process of tracheal mucosal injury is particularly important for the prevention and treatment of benign tracheal stenosis.

The pathogenesis of benign tracheal stenosis is complex and involves various factors, including immunity, inflammation, cell proliferation, differentiation, apoptosis and oxidative stress (3-5). Inflammatory response is an important pathogenic factor (3). Puyo and Dahms (3) demonstrated that patients who underwent tracheal intubations under general anesthesia exhibited ten times the number of polymorphonuclear cells when compared with levels prior to intubation and significantly increased levels of inflammatory factors, including interleukin (IL)-9, IL-6, IL-1 $\beta$ and tumor necrosis factor (TNF), even if tracheal intubation was performed within $3 \mathrm{~h}$. These cytokines and inflammatory factors further aggravate the inflammatory response of the tracheal mucosa, indicating that the pathogenesis of tracheal stenosis following tracheal intubation is associated with the local inflammatory reaction of the trachea and the systemic inflammatory response (3). Following tracheal injury, repeated or sustained inflammatory reactions promote the production of numerous inflammatory factors (such as IL-6, IL-8) and profibrotic cytokines [such as transforming growth factor $\beta 1$ (TGF- $\beta 1$ ), VEGF] involved 
in repair activities, fibroblast cell activation and proliferation, inhibition of apoptosis, extracellular matrix deposition and an imbalance of collagen synthesis and degradation, and, ultimately, excessive proliferation of granulation tissue leading to tracheal stenosis (3-5). Histone deacetylase 2 (HDAC2), a subtype of HDAC closely associated with chronic airway inflammation, primarily regulates the transcriptional expression of inflammatory genes (6). HDAC2 reduces the expression of inflammatory factors IL- 8 and TNF- $\alpha$ by inhibiting the transcription of $\mathrm{NF}-\kappa \mathrm{B}$, improving the prognosis of bronchial asthma and chronic obstructive pulmonary disease $(7,8)$. However, systematic research on the associated between HDAC2 and tracheal stenosis following injury remains unclear.

Penicillin, erythromycin and budesonide are classic anti-inflammatory drugs and have been reported to have therapeutic effects on tracheal stenosis $(9,10)$. Although these drugs are widely used for clinical control of various inflammatory reactions, their mechanisms of action are different. Penicillin is a highly effective, low-toxicity antibiotic with a killing effect on sensitive bacteria (11). Erythromycin is an antibiotic with decent effectiveness when administered orally and is involved with a variety of biological activities, including immune regulation and the inhibition of inflammatory factors (12). Budesonide is effective for non-infectious inflammation; however, it is not ideal for infectious inflammation (13). Which drug is more powerful for protecting against tracheal stenosis following injury remains to be elucidated. Additionally, whether a combination of these drugs to produce a synergistic effect following injury and whether this combination affects the development of tracheal stenosis by regulating HDAC also remains unclear. The current study investigated the effects of different anti-inflammatory drugs on a model of tracheal stenosis following injury, compared their protective effects on tracheal stenosis following injury and explored the possible mechanisms.

\section{Materials and methods}

Experimental animals. A total of 32 New Zealand rabbits (4 weeks old; 16 males and 16 females, $2.5-3 \mathrm{~kg}$ ) were purchased from Nanchang Longping Rabbit Industry Co., Ltd. The rabbits were bred in a specific pathogen-free class barrier system, maintained in a temperature-controlled room $\left(18-22^{\circ} \mathrm{C}\right)$ with 12-h light/dark cycles and permitted to eat and drink freely. The present study followed international, national and institutional guidelines for humane animal treatment and complied with the relevant legislation (14). Experiments were approved by the Animal Experimental Ethics Committee of Guangxi Medical University, Nanning, China with supervision by the facility in which the studies were conducted (approval no. 201806020).

Experimental reagents and instruments. Penicillin sodium (Shandong Lukang Record Pharmaceutical Co., Ltd.) for injection, erythromycin enteric-coated tablets (Yichang Humanwell Pharmaceutical Co., Ltd.), budesonide suspension (AstraZeneca) for inhalation, rabbit anti-collagen I (Col-I) polyclonal antibodies (cat. no. bs-10423R; BIOSS; dilution, 1:400); rabbit anti-collagen III (Col-III) polyclonal antibodies (cat. no. bs-0549R; BIOSS; dilution, 1:400), mouse anti-GAPDH monoclonal antibodies (cat. no. TA-08; dilution, 1:2,000); horseradish-labelled goat anti-rabbit immunoglobulin G
(IgG; H+L) (cat. no. ZB-2301; Beijing Zhongshan Jinqiao Biotechnology Co., Ltd.; dilution, 1:100), rabbit anti-HDAC2 polyclonal antibody (cat. no. bs-1813R; BIOSS; dilution, 1:400), conjugated goat anti-rabbit IgG Cy3, (cat. no. CW0159S; CoWin Biosciences; dilution, 1:200), ready-to-use DAPI staining solution (cat. no. KGA215-50; Nanjing KeyGen Biotech Co., Ltd.), rabbit TGF- $\beta 1$ ELISA kit (cat. no. MM-3684001; MEIMIAN), rabbit vascular endothelial growth factor (VEGF) ELISA kit (cat. no. MM-021001; MEIMIAN), a rabbit IL-8 ELISA kit (cat. no. MM-030101; MEIMIAN), rabbit polyclonal anti-TGF $\beta 1$ (cat. no. bs-0086R; BIOSS; dilution, 1:500-1:2,000), rabbit polyclonal anti-VEGF (cat. no. bs-1313R; BIOSS; dilution, 1:500-1:2,000), rabbit monoclonal anti-IL8 (cat. no. ab34100; Abcam; dilution, 1:1,000); rabbit polyclonal anti-HDAC2 (cat. no. OM105905; Omnimabs; dilution, 1:500-1:2,000), fluorescence microscope (CKX53; Olympus Corporation), microplate reader (RT-6100; Rayto Life and Analytical Sciences Co., Ltd.), protein vertical electrophoresis instrument (DYY-6C; Beijing Liuyi Instrument Factory) and Ultra High Sensitivity Chemiluminescence Imaging System (Chemi-DocTM XRS+; Bio-Rad Laboratories, Inc.) were used in the current study.

Establishment of a tracheal stenosis model and sampling. In total, 30 rabbits were used to establish a tracheal stenosis model, and 2 rabbits were used as normal rabbits to compare with the model to verify that the tracheal stenosis model was successfully established. Modeling was performed according to the method described by Nakagishi et al (15). The rabbits were fasted for $8 \mathrm{~h}$ prior to modeling and anesthetized intravenously with $3 \%$ pentobarbital sodium (cat. no. P11011; Merck KGaA; $30 \mathrm{mg} / \mathrm{kg}$ ) (16). To enhance analgesia, the rabbits were injected with $2 \%$ lidocaine hydrochloride (cat. no. T1144; TargetMol) into the anterior neck. Following anesthesia, the rabbits were placed in the supine position and fixed onto the operating table. The skin in the anterior cervical region was prepared and disinfected twice with $0.5 \%$ iodophor (cat. no. YS0545; Amresco). A longitudinal incision $(\sim 4-5 \mathrm{~cm})$ was made in the skin and the subcutaneous tissues and muscles were separated layer by layer to expose the trachea. An annular tracheotomy was performed in cartilage spaces 3 and 4 . The length of the incision was $2 / 3$ of the circumference of the trachea to avoid injury to the tracheal cartilage. The proximal end of the trachea was lifted to avoid suffocation caused by blood flow back to the distal end of the trachea and bleeding was stopped by compressing the tracheal incision. A rigid nylon brush was inserted into the distal trachea $\sim 1.5 \mathrm{~cm}$ through the incision and rubbed back and forth 20 times on the front and side walls of the trachea. If intratracheal hemorrhage occurred due to friction, a gauze was used for hemostasis. After no obvious hemorrhage, a single no. 4.0 thread was used to carefully suture the trachea layer by layer irregularly over the following 3 layers: i) The muscular layer; ii) the subcutaneous tissue layer and iii) skin. The wound was disinfected and covered with sterile gauze. The rabbits were returned to their cages after waking up naturally.

All rabbits were fasted for $8 \mathrm{~h}$ prior to sampling. After performing anesthesia, as described above, $5 \mathrm{ml}$ blood was collected from rabbit ear veins and centrifuged at 1,400 $\mathrm{x} \mathrm{g}$ for $10 \mathrm{~min}$ at $4^{\circ} \mathrm{C}$ at a centrifugation radius of $11.5 \mathrm{~cm}$. The supernatant was collected and transferred to a cryotube at $-80^{\circ} \mathrm{C}$ for storage. The skin was cut in the anterior cervical region and the 
subcutaneous tissues and muscles were separated layer by layer to expose the trachea. The trachea was transected $2 \mathrm{~cm}$ below the original tracheal incision. The end of the trachea was compressed with pre-prepared gauze to prevent blood from flowing into the distal trachea and causing suffocation and contaminating the collected bronchoalveolar lavage fluid (BALF). A syringe was inserted into the trachea and fixed with a 4.0 surgical suture. Left lung alveolar lavage was performed twice with $10 \mathrm{ml}$ normal saline at $4^{\circ} \mathrm{C}$. At each lavage, BALF was pumped into a centrifuge tube. Right lungs were lavaged twice using the same protocol. The BALF of both lungs was mixed and centrifuged at $1,400 \mathrm{xg}$ for $10 \mathrm{~min}$ at $4^{\circ} \mathrm{C}$ at a centrifugation radius of $11.5 \mathrm{~cm}$. The supernatant was removed and stored in a cryotube at $-80^{\circ} \mathrm{C}$. Once the BALF samples were obtained, the rabbits were sacrificed via an injection of $30 \mathrm{ml}$ air $/ \mathrm{kg}$ into the ear vein (17). The narrow trachea was collected, and tracheal tissue specimens were stored separately at $-80^{\circ} \mathrm{C}$ for relevant testing.

Experimental grouping. Rabbits were randomly divided into 5 groups. All drug doses were based on body surface area calculated using the Meeh-Rubner formula: $\mathrm{A}=\mathrm{K} \times\left(\mathrm{W}^{2 / 3} / 10^{4}\right)$, where $\mathrm{A}$ is body surface area, the constant $\mathrm{K}$ is 10.6 for humans and 10.1 for rabbits $\mathrm{W}$ is weight (18). The groups were as follows: i) The control (CON) group, untreated; ii) the PEN group, animals were treated with penicillin $(43500 \mathrm{U} / \mathrm{kg})$ twice daily via intramuscular injection; iii) the ERY group, animals were treated with erythromycin $(13.6 \mathrm{mg} / \mathrm{kg})$ twice a day administered via gavage; iv) the BUD group, animals were treated with budesonide suspension $(0.05 \mathrm{mg} / \mathrm{kg})$ twice a day via atomization inhalation; and v) the PEN + ERY + BUD group, animals were treated with penicillin $(43,500 \mathrm{U} / \mathrm{kg})$ twice daily via intramuscular injection, erythromycin $(13.6 \mathrm{mg} / \mathrm{kg})$ twice a day via gavage administration and budesonide suspension $(0.05 \mathrm{mg} / \mathrm{kg})$ twice a day via atomization inhalation. The atomization inhalation method used was previously reported by Lei et al (19). These drugs are often used in humans (clinical treatment) and animals (animal experiments) and are relatively safe at these doses and time of use $(11,20)$. To the best of our knowledge, there are no previous reports indicating that these drugs have obvious side effects on rabbit organ function or body weight at these doses and time of use. The results of preliminary experiments did not report obvious side effects of these drugs on organs such as the liver and kidney or body weight. A total of 6 rabbits were included in each group and samples were collected and tested after 10 days of continuous treatment.

Hematoxylin and eosin $(H \& E)$ staining. The tracheal tissues of the normal and model groups were fixed with $4 \%$ paraformaldehyde solution at $25^{\circ} \mathrm{C}$ for $24 \mathrm{~h}$, then rinsed for $6-8 \mathrm{~h}$ and dehydrated with ethanol solutions of 70,80 and $90 \%$ and a mixture of pure alcohol and xylene at $25^{\circ} \mathrm{C}$ for $15 \mathrm{~min}$, xylene I for $15 \mathrm{~min}$ and xylene II for $15 \mathrm{~min}$ until tissues were transparent. Tissues were incubated in a mixture of xylene and paraffin at $25^{\circ} \mathrm{C}$ for $15 \mathrm{~min}$ and then placed in paraffin I followed by paraffin II at $25^{\circ} \mathrm{C}$ for $50-60 \mathrm{~min}$ each. Tissues were paraffin-embedded and sliced (5 $\mu \mathrm{m}$ thick). The paraffinized sections were baked at $45^{\circ} \mathrm{C}$ and Paraffin sections were dewaxed with xylene I followed by xylene II for $5 \mathrm{~min}$ each, then placed in 100, 95, 90, 80 and $70 \%$ alcohol solutions for 3 min each, and then placed in distilled water for $3 \mathrm{~min}$. Once tissues were washed with distilled water, each section was placed in an $0.2 \%$ aqueous solution of hematoxylin at $25^{\circ} \mathrm{C}$ for $3 \mathrm{~min}$ and incubated in an $1 \%$ ethanol differentiation solution at $25^{\circ} \mathrm{C}$ for $15 \mathrm{sec}$, washed slightly with distilled water and returned to the blue hematoxylin solution at $25^{\circ} \mathrm{C}$ for $15 \mathrm{sec}$. Sections were then rinsed with water, stained with $0.5 \%$ eosin at $25^{\circ} \mathrm{C}$ for $3 \mathrm{~min}$ and rinsed with water again. Sections were placed in 95 and 100\% alcohol for 3 min each for dehydration until transparent, sealed and examined by an Olympus CX41 light microscope at x400 magnification.

Immunofluorescence assay. Tissue sections were baked in an oven at $65^{\circ} \mathrm{C}$ for $2 \mathrm{~h}$. Sections were placed into dimethylbenzene (cat. no. X820584; Macklin) for $10 \mathrm{~min}$ and then treated with 100, 100, 95 and $80 \%$ ethanol and purified water for 5 min each time. Antigen retrieval was performed using citrate buffer ( $\mathrm{pH} 6.0$ ) at $121^{\circ} \mathrm{C}$ for $2 \mathrm{~min}$. Sections were blocked with $5 \%$ BSA (Gibco; Thermo Fisher Scientific, Inc.) at $37^{\circ} \mathrm{C}$ for $30 \mathrm{~min}$ and incubated with primary monoclonal anti-HDAC2 antibody (1:400) overnight at $4^{\circ} \mathrm{C}$. Sections were washed with PBS three times and incubated with fluorescent antibodies against $\mathrm{Cy} 3(1: 200)$ at $37^{\circ} \mathrm{C}$ for $30 \mathrm{~min}$. DAPI was added to the tissue slices, which were incubated at $25^{\circ} \mathrm{C}$ for $5 \mathrm{~min}$ in the dark. Excess DAPI was washed away with PBS and sections were washed with water for $1 \mathrm{~min}$. Sections were mounted using an ultra-clean high-grade sealant (cat. no. BA7004; BaSO Biotech Co., Ltd.) and observed with a fluorescence microscope (x400 at magnification). Images were quantitatively analyzed uisng Image J software (version: $1.52 \mathrm{v}$; National Institutes of Health).

Immunohistochemistry. The tracheal tissues were fixed with $4 \%$ paraformaldehyde solution at $25^{\circ} \mathrm{C}$ for $24 \mathrm{~h}$, then embedded in paraffin using a conventional method (21). Tissues were cut into $5-\mu \mathrm{m}$ sections and incubated with $0.3 \%$ endogenous peroxidase blocking solution (cat. no. P0100A; Beyotime Institute of Biotechnology) at $25^{\circ} \mathrm{C}$ for $20 \mathrm{~min}$. Sections were placed into dimethylbenzene (cat. no. X820584; Macklin) for 10 min and then treated with 100, 100, 95 and $80 \%$ ethanol and purified water for $5 \mathrm{~min}$ each time at $25^{\circ} \mathrm{C}$. Sections were then incubated at room temperature for $10 \mathrm{~min}$ with $3 \%$ hydrogen peroxide solution (cat. no. CS-PYJ1138; Shanghai Fusheng Co., Ltd.; https://www.biomart.cn/infosupply/37396991.htm) and washed with PBS three times (3 min each). Antigen retrieval was performed using citrate buffer $(\mathrm{pH} 6.0)$ at $121^{\circ} \mathrm{C}$ for $2 \mathrm{~min}$. Sections were blocked with 5\% BSA (Gibco; Thermo Fisher Scientific, Inc.) at $37^{\circ} \mathrm{C}$ for $30 \mathrm{~min}$ and incubated with a primary polyclonal antibody (anti-collagen I and anti-collagen III) overnight at $4^{\circ} \mathrm{C}$. Following this, sections were incubated with horseradish peroxide-conjugated goat anti-rabbit $\mathrm{IgG}$ for $30 \mathrm{~min}$ at $37^{\circ} \mathrm{C}$, according to the manufacturer's protocol, and mounted with epoxy resin. Tissue sections were observed with an Olympus CX41 light microscope at x400 magnification.

ELISA kits. Levels of IL-8, TGF- $\beta 1$ and VEGF in the serum and BALF of the groups were detected using ELISA kits, according to the manufacturer's protocol. Optical density values at a wavelength of $450 \mathrm{~nm}$ were determined using a microplate reader.

Western blotting. Total proteins were extracted using RIPA cell lysate (cat. no. C1053; Applygen Technologies Inc.) and protein concentrations were determined using bicinchoninic acid assays. 


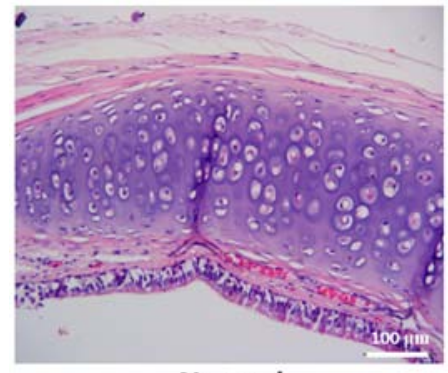

Normal

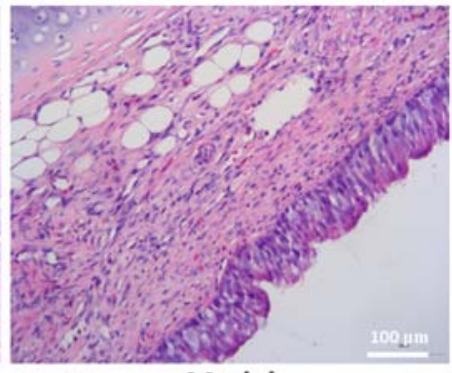

Model

Figure 1. Pathological analysis to determine whether the tracheal stenosis model was successfully established. Scale bar, $100 \mu \mathrm{m}$. Blue-purple staining, nucleus. Red staining, cytoplasm. Normal, untreated normal rabbits. Model, rabbit tracheal stenosis models.
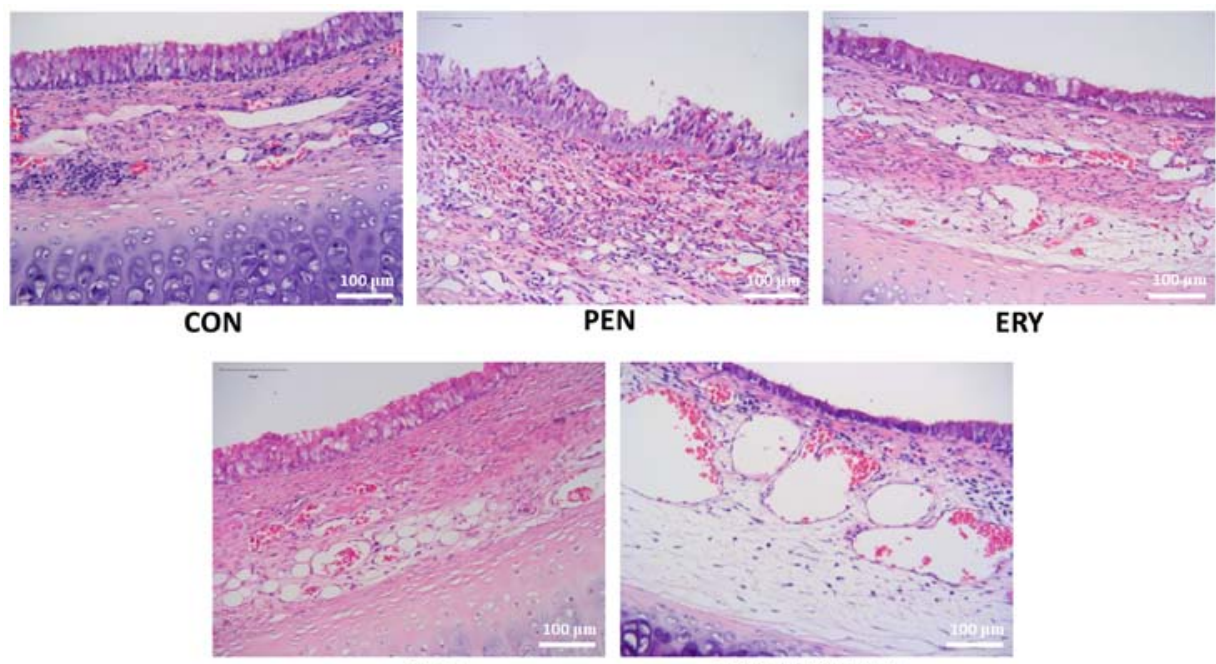

BUD

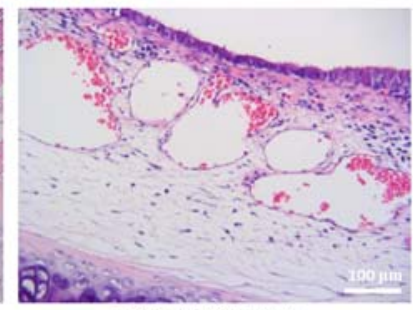

PEN+ERY+BUD

Figure 2. Results for hematoxylin and eosin staining of tracheal tissues in each group. Scale bar, $100 \mu \mathrm{m}$. Blue-purple staining, nucleus. Red staining, cytoplasm. CON, untreated rabbit tracheal stenosis model; PEN, rabbit tracheal stenosis model treated with penicillin; ERY, rabbit tracheal stenosis model treated with erythromycin; BUD, rabbit tracheal stenosis model treated with budesonide; PEN + ERY + BUD, rabbit tracheal stenosis model treated with penicillin, erythromycin and budesonide.

Proteins $(50 \mu \mathrm{g} /$ lane) were separated using $12 \%$ SDS-PAGE and electrotransferred to PVDF membranes (Cytiva). PVDF membranes were rinsed with TBS for 10-15 min and placed in $3 \%$ TBS-Tween-20 (TBS-T) blocking buffer containing 5\% (w/v) skim milk powder at room temperature overnight. Membranes were incubated at room temperature for $2 \mathrm{~h}$ following the addition of an appropriate dilution of primary antibodies (anti-TGF $\beta 1$, anti-VEGF, anti-IL8, anti-HDAC2 and anti-GAPDH). Membranes were then rinsed with TBS-T three times (5-10 $\mathrm{min} / \mathrm{wash})$ and incubated at room temperature for $1 \mathrm{~h}$ with horseradish peroxidase-labelled goat anti-rabbit $\operatorname{IgG}(1: 2,000$; cat. no. ZB-2301; Beijing Zhongshan Jinqiao Biotechnology Co., Ltd.) and goat anti-mouse IgG (H+L) (1:2,000; cat. no. ZB-2305; Zhongshan Jinqiao Biotechnology Co., Ltd.) secondary antibodies diluted with TBS-T containing $0.05 \%$ (w/v) skim milk powder. Membranes were then rinsed three times with TBS-T (5-10 min/wash). Protein bands were detected using an ECL kit (PerkinElmer, Inc.) and quantified based on ratios relative to GAPDH. Quantification was performed using Quantity One software (version 4.6.6, Bio-Rad Laboratories, Inc.).

Statistical analysis. Experiments were performed in triplicate for each group. Data are presented as the mean $\pm \mathrm{SD}$. One-way
ANOVA followed by Tukey's post hoc test was used to analyze differences between groups. All analyses were conducted using SPSS software (version 19.0; IBM Corp.). $\mathrm{P}<0.05$ was considered to indicate a statistically significant difference.

\section{Results}

Successful establishment of the tracheal stenosis model. In the normal rabbits, mucosa, submucosa, goblet cells and basal cells were clearly visible, and the connective tissues of the submucosal fibers were loose. Mucosal epithelial hyperplasia, fibroblast proliferation, thickening of collagen fibers, disordered collagen arrangement and high levels of inflammatory cell infiltration were observed in the model rabbits. Therefore, the model was successfully established (Fig. 1).

$H \& E$ staining demonstrates that erythromycin reduces tracheal mucosal epithelial tissue hyperplasia. Tracheal lumen stenosis, epithelial hyperplasia, fibroblast hyperplasia, thickening of collagen fibers, disordered arrangement and inflammatory cell infiltration were observed in the CON, PEN and BUD groups of models (Fig. 2). In the ERY group of models, the degree of tracheal stenosis was reduced, 


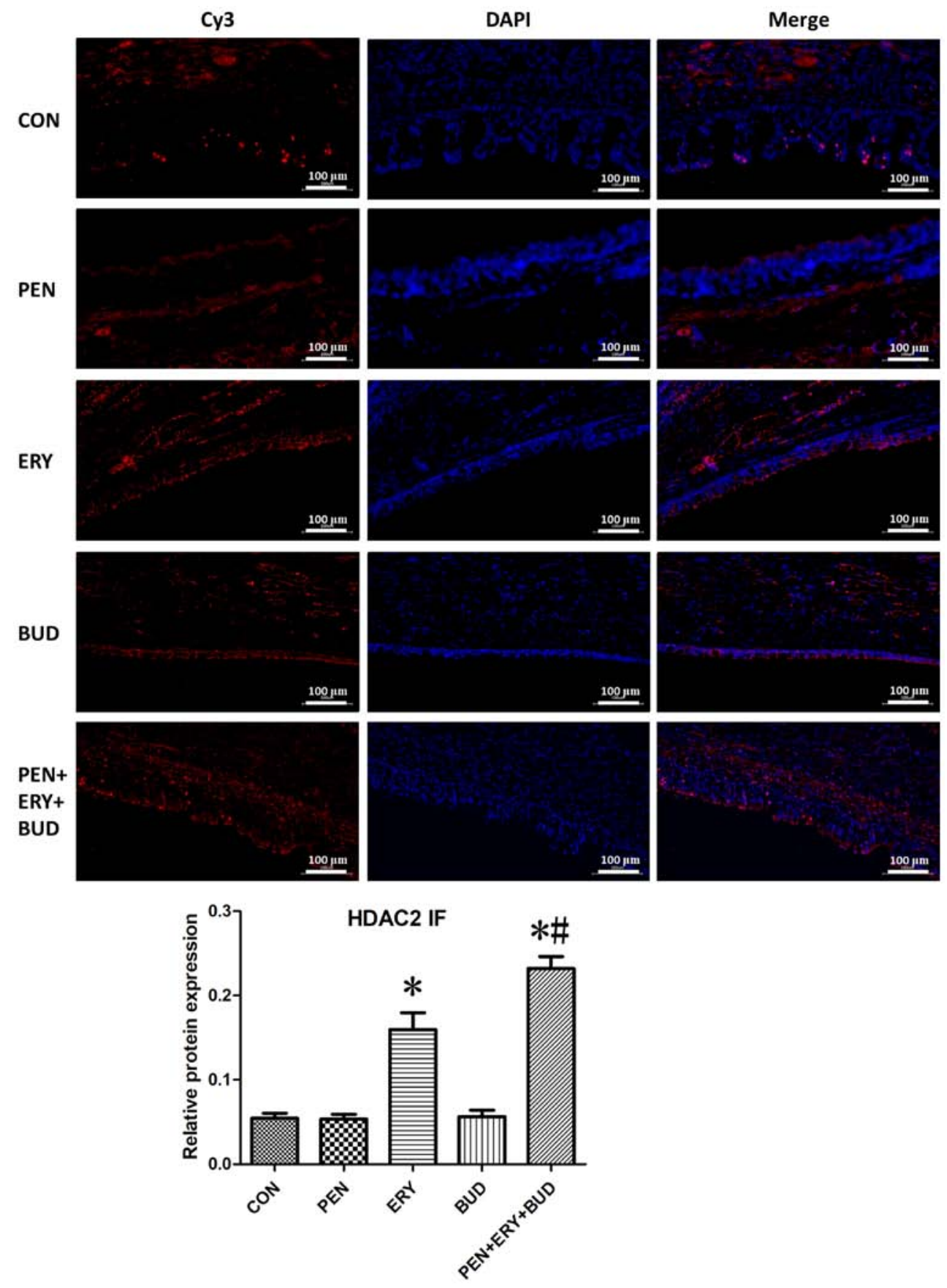

Figure 3. HDAC2 expression in the tracheal tissues of each group was determined by IF. Scale bar, $100 \mu \mathrm{m}$. Red fluorescence (Cy3 staining) indicates the target protein HDAC2 and blue fluorescence (DAPI staining) indicates the nucleus. Data are presented as the mean \pm standard deviation. ${ }^{*} \mathrm{P}<0.05$ vs. CON. ${ }^{\#} \mathrm{P}<0.05$ vs. ERY. HDAC2, histone deacetylase 2; IF, immunofluorescence; CON, untreated rabbit tracheal stenosis model; PEN, rabbit tracheal stenosis model treated with penicillin; ERY, rabbit tracheal stenosis model treated with erythromycin; BUD, rabbit tracheal stenosis model treated with budesonide; PEN + ERY + BUD, rabbit tracheal stenosis model treated with penicillin, erythromycin and budesonide.

mucosal epithelial hyperplasia was alleviated, and fibroblast proliferation and inflammatory cell infiltration were markedly reduced. The effect of the drug treatment in the PEN + ERY + BUD group of models was more obvious compared with the ERY group, the mucosal epithelial hyperplasia was further improved, and the proliferation of fibroblasts was further reduced. Additionally, compared with the PEN and BUD groups, mucosal epithelial hyperplasia in the PEN + ERY + BUD group was significantly relieved, and fibroblast proliferation and inflammatory cell infiltration were significantly reduced (Fig. 2).
Immunofluorescence reveals that erythromycin inhibits the expression of HDAC2 in tracheal tissues. As shown in Fig. 3, red fluorescence (Cy3 staining) indicates the target protein HDAC2 and blue fluorescence (DAPI) indicates the nuclei. Analysis of the tracheal tissues indicated that HDAC2 protein expression in the ERY and PEN + ERY + BUD groups was significantly increased compared with the CON group $(\mathrm{P}<0.05)$. Additionally, HDAC2 expression in the PEN + ERY + BUD group was significantly further increased compared with the ERY group $(\mathrm{P}<0.05)$. There were no differences in HDAC2 protein expression between the PEN, BUD and CON groups. 

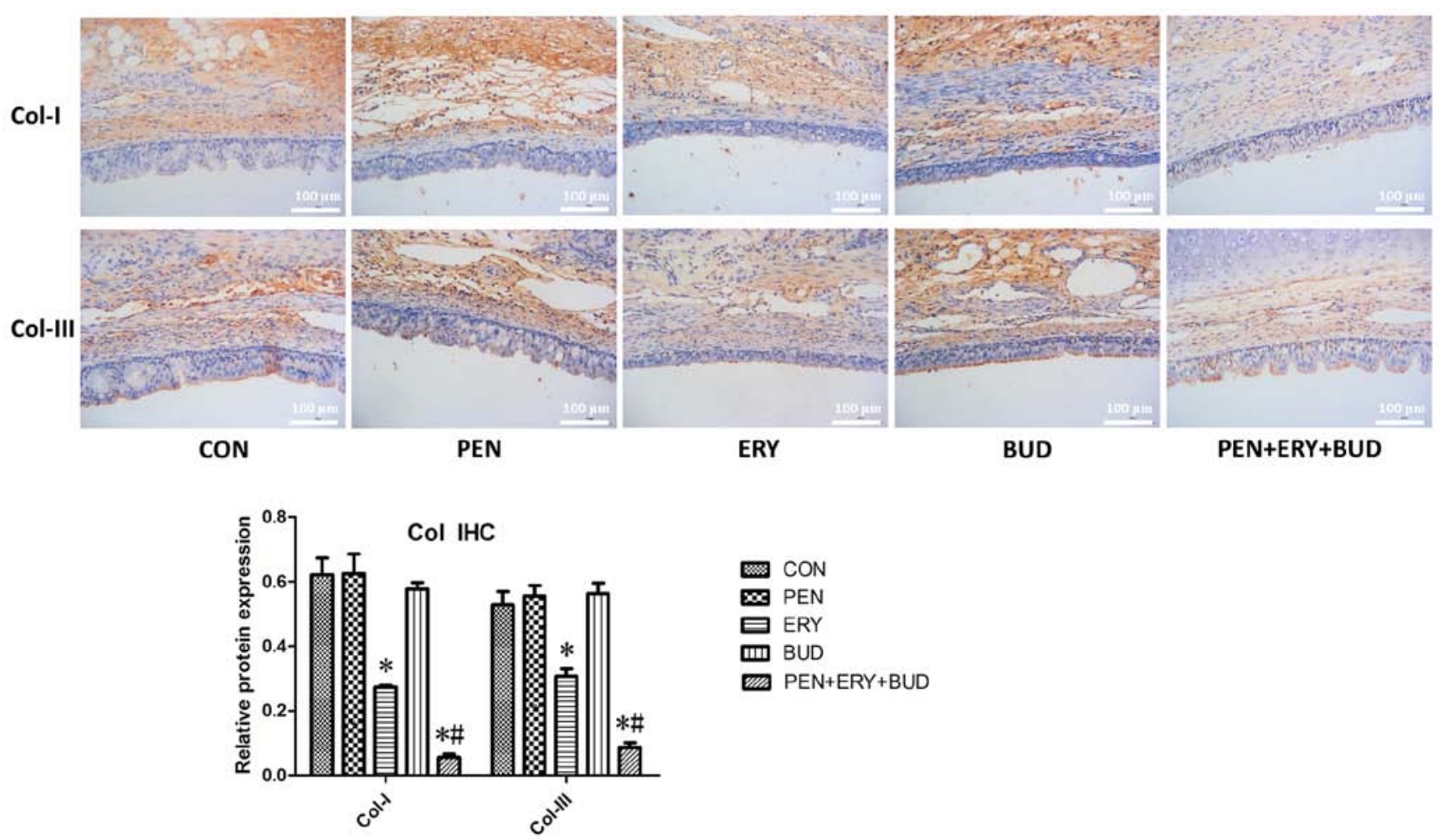

Figure 4. Col-I and Col-III expression in the tracheal tissues of each group was determined by IHC. Scale bar, $100 \mu \mathrm{m}$. Brown staining, target proteins Col-I and Col-III. Blue staining, nucleus. Data are presented as the mean \pm standard deviation. ${ }^{*} \mathrm{P}<0.05$ vs. CON. " $\mathrm{P}<0.05$ vs. ERY. Col-I, collagen I; Col-III, collagen III; IHC, immunohistochemistry; CON, untreated rabbit tracheal stenosis model; PEN, rabbit tracheal stenosis model treated with penicillin; ERY, rabbit tracheal stenosis model treated with erythromycin; BUD, rabbit tracheal stenosis model treated with budesonide; PEN + ERY + BUD, rabbit tracheal stenosis model treated with penicillin, erythromycin and budesonide.

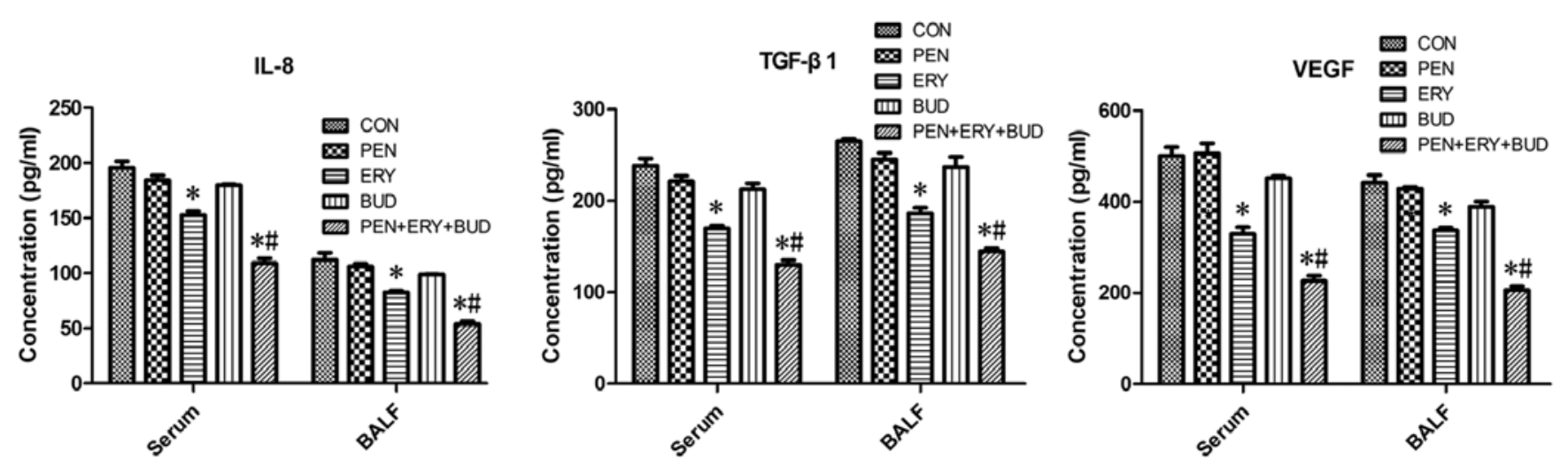

Figure 5. IL-8, TGF- $\beta 1$ and VEGF levels in the serum and BALF of each group were determined by ELISAs. Data are presented as the mean \pm standard deviation in the corresponding histograms. "P<0.05 vs. CON. " $\mathrm{P}<0.05$ vs. ERY. IL-8, interleukin 8; TGF- $\beta 1$, transforming growth factor $\beta 1$; VEGF, vascular endothelial growth factor; BALF, bronchoalveolar lavage fluid; CON, untreated rabbit tracheal stenosis model; PEN, rabbit tracheal stenosis model treated with penicillin; ERY, rabbit tracheal stenosis model treated with erythromycin; BUD, rabbit tracheal stenosis model treated with budesonide; PEN + ERY + BUD, rabbit tracheal stenosis model treated with penicillin, erythromycin and budesonide.

Immunohistochemistry reveals that erythromycin reduces the expression of Col-I and Col-III in tracheal tissue. As shown in Fig. 4, brown staining indicates collagen expression and blue staining indicates the nuclei. In the tracheal tissues, the expression levels of Col-I and Col-III in the ERY and PEN + ERY + BUD groups were significantly lower compared with the CON group $(\mathrm{P}<0.05)$. Moreover, levels in the PEN + ERY + BUD group were significantly further reduced compared with the ERY group $(\mathrm{P}<0.05)$, indicating that fibroblast hyperplasia was controlled and alleviated.
There were no differences in the expression levels of Col-I and Col-III between the PEN, BUD and CON groups.

ELISA indicates that erythromycin reduces the expression of IL-8,TGF- $\beta 1$ and VEGF in serum and BALF. As shown in Fig. 5, the expression levels of IL-8, TGF- $\beta 1$ and VEGF were significantly decreased in the ERY and PEN + ERY + BUD groups compared with the CON group in serum and BALF samples $(\mathrm{P}<0.05)$. Furthermore, levels in the PEN + ERY + BUD group were significantly further 

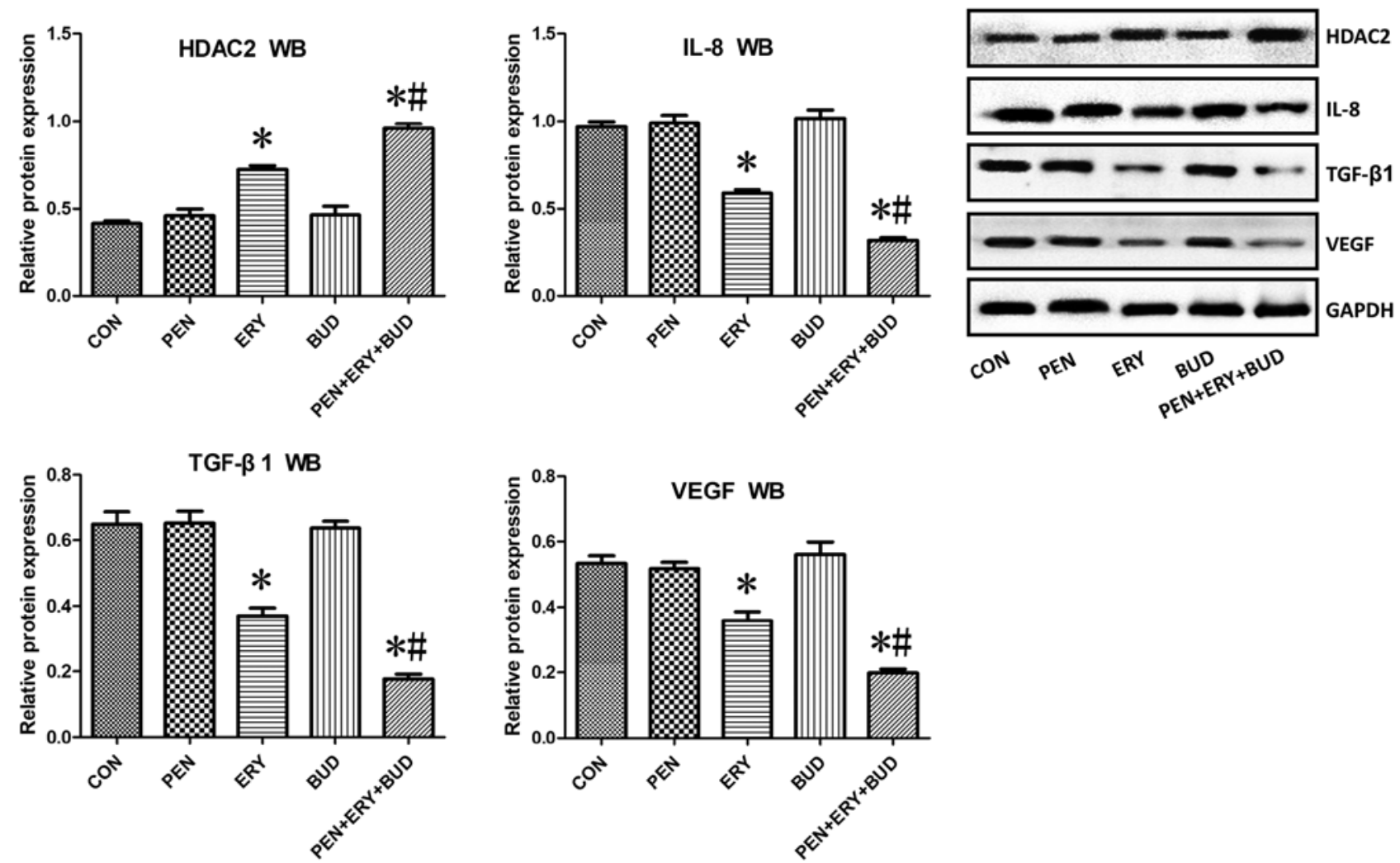

Figure 6. HDAC2, IL-8, TGF- $\beta 1$ and VEGF expression in the tracheal tissue of each group determined by WB. Results were normalized to the internal control GAPDH. Data are presented as the mean \pm standard deviation. ${ }^{*} \mathrm{P}<0.05$ vs. CON. ${ }^{\prime \prime} \mathrm{P}<0.05$ vs. ERY. HDAC2, histone deacetylase 2; IL-8, interleukin 8 ; TGF- $\beta 1$, transforming growth factor $\beta 1$; VEGF, vascular endothelial growth factor; WB, western blotting; CON, untreated rabbit tracheal stenosis model; PEN, rabbit tracheal stenosis model treated with penicillin; ERY, rabbit tracheal stenosis model treated with erythromycin; BUD, rabbit tracheal stenosis model treated with budesonide; PEN + ERY + BUD, rabbit tracheal stenosis model treated with penicillin, erythromycin and budesonide.

reduced compared with the ERY group $(\mathrm{P}<0.05)$. There were no differences in the expression levels of IL-8, TGF- $\beta 1$ and VEGF between the PEN, BUD and CON groups.

Western blotting shows that erythromycin increases the expression of HDAC2 in tracheal tissues and decreases the expression of $I L-8, T G F-\beta 1$ and VEGF. In tracheal tissues, HDAC2 protein expression in the ERY and PEN + ERY + BUD groups was significantly increased compared with the CON group (Fig. 6; $\mathrm{P}<0.05$ ). Additionally, expression in the PEN + ERY + BUD group was significantly further increased compared with the ERY group $(\mathrm{P}<0.05)$. The protein expression of IL-8, TGF- $\beta 1$ and VEGF in the ERY and PEN + ERY + BUD groups were significantly decreased compared with the CON group $(\mathrm{P}<0.05)$ and the expression in the PEN + ERY + BUD group was further reduced compared with the ERY group $(\mathrm{P}<0.05)$. There were no differences in the protein expression of HDAC2, IL-8, TGF- $\beta 1$ or VEGF between the PEN, BUD and CON groups.

\section{Discussion}

There are numerous causes of acquired benign airway stenosis, including traumatic, infectious and inflammatory causes, and benign and idiopathic tumors. In cases of tracheal stenosis following injury, stenosis caused by trauma and iatrogenic factors are the most common types $(1,22)$. Airway stenosis following tracheotomy and tracheal intubation is the most common iatrogenic airway injury (23). Additionally, trauma and anastomosis of the tracheobronchial ends following surgery are common causes. Physical and chemical damage (acidic and alkaline toxic agents), inhalation injury, radiation injury, bronchoscopy intervention airway thermal ablation (laser, argon plasma coagulation, electrocoagulation) are also important causes (24). Compared with malignant airway stenosis, benign airway stenosis is more difficult to treat and more likely to cause long-term complications (1). Furthermore, due to the extended survival of patients, patients and their families have high expectations for the quality of life and the serious short-term and long-term complications caused by surgery are difficult to accept (1). Therefore, the treatment of benign airway stenosis is a difficult issue in the field of respiratory diseases. Presently, transbronchoscopic intervention therapy has gradually become one of the main methods for the treatment of benign airway stenosis (25). However, due to the inevitable secondary airway injury during the treatment of airway stenosis, restenosis following endoscopic intervention therapy is a challenge in the field of interventional respiratory disease (1).

The current methods for the prevention and treatment of tracheobronchial stenosis and restenosis following endoscopic intervention mainly include local application of mitomycin C (26,27), intracavitary brachytherapy (28), drug-coated stents (29,30), balloon dilation (31), argon plasma coagulation (32), cryotherapy (33) and adequate anti-infection treatment (34). Previous studies have confirmed that the 
formation of granulation tissue following tracheal reconstruction and stent implantation is closely associated with an increase in bacterial infections and common pathogens include Pseudomonas aeruginosa, Staphylococcus aureus, Streptococcus viridans, Haemophilus influenzae and Neisseria (34-36). Brown and Montgomery (34) demonstrated that the use of antibiotics significantly reduced the formation of intratracheal granulation tissue following silicone stent implantation. The results indicated that antibiotic therapy may have a preventive effect on tracheal stenosis following tracheal injury. Penicillin is an important $\beta$-lactam antibiotic with high efficiency, low toxicity and wide clinical applications (11). The development of this antibiotic introduced a new era of antibiotic treatment for infectious diseases (11). In the current study, tracheal mucosal epithelial hyperplasia and fibroblast proliferation were not alleviated in the PEN group compared with the CON group. Furthermore, collagen synthesis was not significantly reduced, indicating that infection is not the only factor in tracheal stenosis following injury and is not necessarily the main factor. At the same time, in this study, the tracheal stenosis model was established under strict aseptic conditions; thus reducing the risk of infection by pathogenic microorganisms. Therefore, the therapeutic effect of penicillin on tracheal stenosis after injury in animal models is not as obvious as in clinical applications (5).

Glucocorticoids are commonly used anti-inflammatory and immunosuppressive drugs that bind to the glucocorticoid receptor on the cell membrane and enter the nucleus to regulate the transcription of various genes, thereby controlling cell function (37). The mechanism by which glucocorticoids inhibit scar formation remains unclear. Additionally, whether topical application of glucocorticoids for benign scar stenosis in the central airway is effective is unclear. Yokoi et al (38) reported that inhaled budesonide effectively treated tracheal granulation tissue hyperplasia in patients who underwent tracheostomies following congenital tracheal stenosis repair. The results of the current study demonstrated that tracheal mucosal epithelial hyperplasia and fibroblast proliferation were not significantly improved in the BUD group and that collagen synthesis was not significantly reduced compared with the CON group, indicating that a single inhaled BUD cannot reduce tracheal stenosis following tracheal injury. This is most likely due tracheal stenosis following injury being caused by numerous factors (1). While budesonide reduces non-infectious inflammation, it cannot address inflammation caused by infection and may aggravate infection (39).

In addition to its antibacterial action, erythromycin is a macrolide antibiotic with strong anti-inflammatory and immunomodulatory effects (12). Erythromycin is widely used in chronic inflammatory airway diseases, including asthma, bronchiectasis and diffuse panbronchiolitis, due of its dose-dependent, anti-inflammatory, immunomodulatory, anti-fibroblast proliferative and inhibitory effects on the synthesis and secretion of fibrosis-related factors (40). A previous study confirmed that erythromycin enhanced the activity of HDAC2 in monocytes under oxidative stress, thereby reducing the expression of $\mathrm{NF}-\kappa \mathrm{B}$ and IL-8 (41). Long-term application of low-dose erythromycin exerted an airway anti-inflammatory effect, which reduces the number of acute episodes of chronic obstructive pulmonary disease
(COPD) (42). In the current study, tracheal mucosal epithelial hyperplasia and fibroblast proliferation were significantly improved in the ERY group and collagen synthesis was significantly reduced compared with the $\mathrm{CON}$ group, indicating that erythromycin inhibited the proliferation of tracheal granulation tissue and had preventive and protective effects in tracheal stenosis following injury. Furthermore, the tracheal granulation hyperplasia in the PEN + ERY + BUD group was further inhibited compared with the ERY group, indicating that the drug combination exhibited synergistic effects.

The activation and release of inflammatory cells and inflammatory mediators are conducted by the transcription and regulation of inflammatory genes (8). Histone acetylation and deacetylation are the 'switches' which regulate the transcription of inflammatory genes (8). HDACs remove acetyl groups from the tails of histones and inhibit the transcription of inflammatory genes (6). HDAC2, a subtype of type I HDAC, is located in the nucleus and is primarily involved in inflammatory inhibition $(43,44)$. Previous studies have demonstrated that HDAC2 activity in lung inflammatory cells is reduced by tobacco smoke, leading to transcriptional activation of $\mathrm{NF}-\kappa \mathrm{B}$, which enhances the expression and activity of inflammatory factors IL- 8 and TNF- $\alpha(45,46)$. In chronic airway inflammatory diseases, including asthma and COPD, HDAC2 activity is significantly reduced and is closely associated with disease progression $(7,8)$. This activity can be restored by inhaled corticosteroid therapy (47). Previous studies have reported that low concentrations of theophylline and erythromycin upregulated the protein expression of HDAC2, enhanced HDAC2 activity $(48,49)$ and improved the prognosis of COPD (50). Additionally, erythromycin may increase the activity of HDAC2 by inhibiting the phosphoinositide 3-kinase/protein kinase $\mathrm{B}$ pathway and reducing the release of inflammatory factors, including IL-8, thereby increasing the anti-inflammatory effect of budesonide (51). IL-8 is an important pro-inflammatory signal that promotes the migration of neutrophils to the wound site early in wound healing, alters the local immune environment and initiates granulation hyperplasia for wound repair (52). A previous study on biomarkers that predict tracheal stenosis caused by metal stent implantation demonstrated that elevated IL-8 expression levels were predictive of the development of tracheal stenosis in rabbits (53). Furthermore, a previous study using a murine COPD model revealed that carbocisteine improved airway remodeling, including airway epithelium, smooth muscle thickness, airway fibrosis and decreased levels of $\alpha$-smooth muscle actin and TGF- $\beta 1$, by increasing HDAC 2 expression and activity (54). In the current study, tracheal mucosal epithelial hyperplasia and fibroblast hyperplasia were not improved and there was no significant reduction in collagen synthesis in the PEN and BUD groups compared with the CON group, which was consistent with the lack of a significant upregulation effect of PEN or BUD on the expression of HDAC2 in tracheal tissues. However, erythromycin upregulated the expression of HDAC2 and inhibited hyperplasia in tracheal granulation tissues. Although erythromycin may improve tracheal stenosis following injury through a combination of multiple signaling pathways, this study hypothesized that HDAC2 may represent an important signaling pathway in the development of tracheal stenosis. 
Granulation tissue in tracheal stenosis is characterized by increased angiogenesis and extracellular matrix deposition (5). TGF- $\beta 1$ is a potent extracellular matrix inducer, a chemotactic mediator of fibroblasts and polymorphonuclear cells $(55,56)$ and a fibroblast mitogen (57) that serves an important role in epithelial cell regeneration, fibroblast proliferation and tracheal wound healing following mechanical injury (55-57). VEGF is the most potent specific endothelial cell mitogen and serves a crucial role in stimulating endothelial cell mitosis, migration and angiogenesis $(58,59)$. The production of VEGF indicates the formation of new blood vessels, which is characteristic of tissue repair following injury (5). TGF- $\beta 1$ enhances the repair and reconstruction of tissues by inducing the release of VEGF (60). Lee et al (61) reported that the expression of TGF- $\beta 1$ and VEGF and the number of fibroblasts increased in tracheal granulation tissues obtained by interventional bronchoscopy following tracheostomy. Furthermore, the submucosal layer of the granulation tissues exhibited a further increase in the expression of TGF- $\beta 1$ and VEGF and the number of fibroblasts compared with the epithelial layer and small blood vessels in the submucosa of the granulation tissues were markedly increased (61). Low concentrations of erythromycin $(1 \mathrm{mg} / \mathrm{ml})$, rather than dexamethasone, inhibited TGF- $\beta 1$-induced VEGF production (61). This result is consistent with the inhibitory effects of erythromycin on TGF- $\beta 1$ and VEGF expression in the tracheal granulation tissues observed in the current study.

In the current study, HDAC2, IL- $8, \mathrm{TGF}-\beta 1$ and VEGF expression in the stenotic tracheal tissues of the PEN and BUD groups were not significantly altered compared with the CON group and there were no significant differences in IL-8, TGF- $\beta 1$ and VEGF expression in serum and BALF samples. HDAC2 expression in the stenotic tracheal tissues of the ERY group was increased, the expression of IL- 8 , TGF- $\beta 1$ and VEGF was decreased and the expression of IL- 8 and TGF- $\beta 1$ and VEGF in the serum and BALF was decreased compared with the CON group. Furthermore, HDAC2 expression in the stenotic tracheal tissue was further increased, the expression of IL-8, TGF- $\beta 1$ and VEGF was further decreased and the expression of IL-8, TGF- $\beta 1$ and VEGF in the serum and BALF was significantly decreased in the PEN + ERY + BUD group compared with the ERY group. These results indicated that erythromycin may inhibit the tracheal local and systemic inflammatory response by upregulating HDAC2, promoting the downregulation of TGF- $\beta 1$ and VEGF, inhibiting the proliferation of fibroblasts and the synthesis and secretion of fibrosis-related factors, reducing the synthesis of Col-I and Col-III, limiting excessive repair of the damaged trachea and protecting against tracheal stenosis following injury. The combined use of erythromycin, budesonide and penicillin produced a synergistic effect and protective effects are further enhanced.

In conclusion, it is challenging to prevent tracheal stenosis following injury and treatment efficacy is poor due to a combination of various factors such as infection, inflammation and oxidative stress (1). The use of erythromycin alone significantly inhibited the proliferation of tracheal granulation tissues and improved tracheal stenosis following injury, while penicillin and budesonide had no significant effect. Erythromycin upregulated the expression of HDAC2, enhanced anti-inflammatory effect, downregulated the expression of TGF- $\beta 1$, VEGF, Col-I and Col-III and inhibited excessive tissue repair, thereby effectively reducing tracheal stenosis following tracheal injury. The combined use of penicillin, erythromycin and budesonide produced a synergistic effect, which may further upregulate the expression of HDAC2, inhibit local airway and systemic inflammatory responses, inhibit granulation hyperplasia and enhance the protective effect against tracheal stenosis following injury. The main purpose of this study was to compare the therapeutic effect of penicillin, erythromycin and budesonide alone on tracheal stenosis after injury, and to make a preliminary understanding on the effect of combined drug treatment Due to the limitation of experimental time and funding, future studies on the effects of $\geq 2$ drug combinations will be conducted. For the stability of the experiment, HDAC2 activators and inhibitors were selected to initially verify the theory in this study. Future studies will consider gene knock-in or knock-out experiments from the perspective of genes to further verify the theory.

\section{Acknowledgements}

Not applicable.

\section{Funding}

The current study was supported by a grant from the National Nature Science Foundation of China (grant no. 81760001).

\section{Availability of data and materials}

The datasets used and/or analyzed during the current study are available from the corresponding author on reasonable request.

\section{Authors' contributions}

$\mathrm{ZH}$ and GL designed the experiments. ZH, PW, LG and WL performed the experiments. LG, WL, TZ, CQ and ZC analyzed the data and $\mathrm{ZH}$ validated the analysis. $\mathrm{ZH}$ prepared the manuscript and GL revised the manuscript. All authors read and approved the final manuscript.

\section{Ethics approval and consent to participate}

The current study was approved by the Animal Experimental Ethics Committee of Guangxi Medical University, Nanning, China.

\section{Patient consent for publication}

Not applicable.

\section{Competing interests}

The authors declare that they have no competing interests.

\section{References}

1. Su ZQ, Wei XQ, Zhong CH, Chen XB, Luo WZ, Guo WL, Wang YZ and Li SY: The cause and efficacy of benign tracheal stenosis. Zhonghua Jie He He Hu Xi Za Zhi 36: 651-654, 2013 (In Chinese). 
2. Zhang J, Wang T, Wang J,Pei YH, Xu M, Wang YL, Zhang X and Wang C: Effect of three interventional bronchoscopic methods on tracheal stenosis and the formation of granulation tissues in dogs. Chin Med J (Engl) 123: 621-627, 2010.

3. Puyo CA and Dahms TE: Innate immunity mediating inflammation secondary to endotracheal intubation. Arch Otolaryngol Head Neck Surg 138: 854-858, 2012.

4. Lawrence DA, Branson B, Oliva I and Rubinowitz A: The wonderful world of the windpipe: A review of central airway anatomy and pathology. Can Assoc Radiol J 66: 30-43, 2015.

5. Li LH, Xu MP, Gan LM, Li Y, Liang YL, Li WT, Qin EY, Gan JH and Liu GN: Effect of low dose erythromycin on the proliferation of granulation tissue after tracheal injury. Zhonghua Yi Xue Za Zhi 97: 777-781, 2017 (In Chinese).

6. Yao H and Rahman I: Current concepts on oxidative/carbonyl stress, inflammation and epigenetics in pathogenesis of chronic obstructive pulmonary disease. Toxicol Appl Pharmacol 254: $72-85,2011$

7. Ito K, Lim S, Caramori G, Chung KF, Barnes PJ and Adcock IM: Cigarette smoking reduces histone deacetylase 2 expression, enhances cytokine expression, and inhibits glucocorticoid actions in alveolar macrophages. FASEB J 15: 1110-1112, 2001.

8. Barnes PJ: Role of HDAC2 in the pathophysiology of COPD. Annu Rev Physiol 71: 451-464, 2009.

9. Croft CB, Zub K and Borowiecki B: Therapy of iatrogenic subglottic stenosis: A steroid/antibiotic regimen. Laryngoscope 89: 482-489, 1979.

10. Zhou L, Li Y, Gan LM, Qin EY, Meng XY, Gan JH, Li WT, Qin CC and Liu GN: Effects of different drugs on bronchial stenosis by TGF- $\beta /$ mTOR signaling pathway in rabbit model. Zhonghua Yi Xue Za Zhi 99: 1898-1903, 2019 (In Chinese).

11. Thompson SR III: Treatment of syphilis in pregnancy. J Am Vener Dis Assoc 3: 158-167, 1976.

12. Giamarellos-Bourboulis EJ: Macrolides beyond the conventional antimicrobials: A class of potent immunomodulators. Int J Antimicrob Agents 31: 12-20, 2008

13. Stern A, Skalsky K, Avni T, Carrara E, Leibovici L and Paul M Corticosteroids for pneumonia. Cochrane Database Syst Rev 12 CD007720, 2017.

14. Kilkenny C, Browne WJ, Cuthill IC, Emerson M and Altman DG Improving bioscience research reporting: The ARRIVE guidelines for reporting animal research. J Pharmacol Pharmacother 1: 94-99, 2010.

15. Nakagishi Y, Morimoto Y, Fujita M, Ozeki Y, Maehara T and Kikuchi M: Rabbit model of airway stenosis induced by scraping of the tracheal mucosa. Laryngoscope 115: 1087-1092, 2005 .

16. White WJ and Field KJ: Anesthesia and surgery of laboratory animals. Vet Clin North Am Small Anim Pract 17: 989-1017, 1987.

17. Harken AH, Lillo RS and Haut MJ: The depressant influence of extracellular fluid hyperoxia on liver slice oxygen uptake. J Lab Clin Med 89: 1269-1277, 1977.

18. Spiers DE and Candas V: Relationship of skin surface area to body mass in the immature rat: A reexamination. $\mathrm{J}$ Appl Physiol Respir Environ Exerc Physiol 56: 240-243, 1984.

19. Lei H, Qian GS, Wu GM and Huang GJ: Transfection of human beta defensin 2 gene into the lung by aerosol inhalation: Experiment with rats. Zhonghua Yi Xue Za Zhi 88: 1425-1428, 2008 (In Chinese)

20. Lin JT, Zhang YM, Zhou X, Wang CZ, Huang M, Liu CT, Wu CG, Wan HY, Yu WC and Dai YR: Chinese expert consensus for non-antiinfective effects and clinical use of macrolides. Zhonghua Nei Ke Za Zhi 56: 546-557, 2017 (In Chinese).

21. Ushida K, Asai N, Uchiyama K, Enomoto A and Takahashi M: Development of a method to preliminarily embed tissue samples using low melting temperature fish gelatin before sectioning: A technical note. Pathol Int 68: 241-245, 2018

22. Stauffer JL, Olson DE and Petty TL: Complications and consequences of endotracheal intubation and tracheotomy. A prospective study of 150 critically ill adult patients. Am J Med 70: 65-76, 1981

23. Plojoux J, Laroumagne S, Vandemoortele T, Astoul PJ, Thomas PA and Dutau H: Management of benign dynamic 'A-shape' tracheal stenosis: A retrospective study of 60 patients. Ann Thorac Surg 99: 447-453, 2015.

24. Barros CD, Fernandez-Bussy S, Folch E, Flandes AJ and Majid A: Non-malignant central airway obstruction. Arch Bronconeumol 50: 345-354, 2014 (In English, Spanish).
25. Zhang J, Wang J, Wang T, Xu M, Dang BW, Pei YH and Zhang CY: A pilot study on interventional bronchoscopy in the management of airway stenosis with benign hyperplasia. Zhonghua Jie $\mathrm{He} \mathrm{He}$ Hu Xi Za Zhi 34: 334-338, 2011 (In Chinese).

26. Ferreira S, Nogueira C, Oliveira A, Neves S, Almeida J and Moura e Sá J: Bronchoscopic dilation techniques and topical application of mitomycin- $\mathrm{C}$ in the treatment of tracheal stenosis post intubation-two case reports. Rev Port Pneumol 16: 149-156, 2010 (In Portuguese).

27. Simpson CB and James JC: The efficacy of mitomycin-C in the treatment of laryngotracheal stenosis. Laryngoscope 116: $1923-1925,2006$

28. Tendulkar RD, Fleming PA, Reddy CA, Gildea TR, Machuzak M and Mehta AC: High-dose-rate endobronchial brachytherapy for recurrent airway obstruction from hyperplastic granulation tissue. Int J Radiat Oncol Biol Phys 70: 701-706, 2008.

29. Zhu GH, Ng AH, Venkatraman SS, Boey FYC, Wee ALY, Trasti SL and Lim LHY: A novel bioabsorbable drug-eluting tracheal stent. Laryngoscope 121: 2234-2239, 2011

30. Shin JH, Song HY, Seo TS, Yuk SH, Kim YH, Cho YM, Choi GB, Kim TH and Suh JY: Influence of a dexamethasone-eluting covered stent on tissue reaction: An experimental study in a canine bronchial model. Eur Radiol 15: 1241-1249, 2005.

31. Venhaus M, Behn C, Freitag L and Zimmermann K: Simulations and experiments of the balloon dilatation of airway stenoses. Biomed Tech (Berl) 54: 187-195, 2009.

32. Erelel M, Yakar F and Yakar A: Endobronchial tuberculosis with lobar obstruction successfully treated by argon plasma coagulation. South Med J 102: 1078-1081, 2009.

33. Krimsky WS, Rodrigues MP, Malayaman N and Sarkar S: Spray cryotherapy for the treatment of glottic and subglottic stenosis. Laryngoscope 120: 473-477, 2010.

34. Brown MT and Montgomery WW: Microbiology of tracheal granulation tissue associated with silicone airway prostheses. Ann Otol Rhinol Laryngol 105: 624-627, 1996.

35. Simoni P and Wiatrak BJ: Microbiology of stents in laryngotracheal reconstruction. Laryngoscope 114: 364-367, 2004.

36. Matt BH, Myer CR III, Harrison CJ, Reising SF and Cotton RT: Tracheal granulation tissue. A study of bacteriology. Arch Otolaryngol Head Neck Surg 117: 538-541, 1991.

37. Barnes PJ: How corticosteroids control inflammation: Quintiles Prize Lecture 2005. Br J Pharmacol 148: 245-254, 2006.

38. Yokoi A, Nakao M, Bitoh Y, Arai H, Oshima Y and Nishijima E: Treatment of postoperative tracheal granulation tissue with inhaled budesonide in congenital tracheal stenosis. J Pediatr Surg 49: 293-295, 295, 2014.

39. Toren K, Blanc PD, Qvarfordt I, Aspevall O and Schioler L: Inhaled corticosteroids use and risk of invasive pneumococcal disease risk in a population-based study. Ann Am Thorac Soc 17: 1570-1575, 2020

40. Amsden GW: Anti-inflammatory effects of macrolides-an underappreciated benefit in the treatment of community-acquired respiratory tract infections and chronic inflammatory pulmonary conditions? J Antimicrob Chemother 55: 10-21, 2005.

41. Li M,Zhong X, He Z, Wen M, Li J, Peng X, Liu G, Deng J, Zhang J and Bai J: Effect of erythromycin on cigarette-induced histone deacetylase protein expression and nuclear factor-kB activity in human macrophages in vitro. Int Immunopharmacol 12: 643-650, 2012

42. Seemungal TA, Wilkinson TM, Hurst JR, Perera WR, Sapsford RJ and Wedzicha JA: Long-term erythromycin therapy is associated with decreased chronic obstructive pulmonary disease exacerbations. Am J Respir Crit Care Med 178: 1139-1147, 2008.

43. Zhang Q, Zhao K, Shen Q, Han Y, Gu Y, Li X, Zhao D, Liu Y, Wang C, Zhang X, et al: Tet2 is required to resolve inflammation by recruiting Hdac2 to specifically repress IL-6. Nature 525 : 389-393, 2015.

44. Ito K, Yamamura S and Essilfie-Quaye S, Cosio B, Ito M, Barnes PJ and Adcock IM: Histone deacetylase 2-mediated deacetylation of the glucocorticoid receptor enables NF-kappaB suppression. J Exp Med 203: 7-13, 2006.

45. Adenuga D, Yao H, March TH, Seagrave J and Rahman I: Histone deacetylase 2 is phosphorylated, ubiquitinated, and degraded by cigarette smoke. Am J Respir Cell Mol Biol 40: 464-473, 2009.

46. Barnes PJ: Immunology of asthma and chronic obstructive pulmonary disease. Nat Rev Immunol 8: 183-192, 2008.

47. Ito K, Caramori G, Lim S, Oates T, Chung KF, Barnes PJ and Adcock IM: Expression and activity of histone deacetylases in human asthmatic airways. Am J Respir Crit Care Med 166: 392-396, 2002 
48. Cosio BG, Tsaprouni L, Ito K, Jazrawi E, Adcock IM and Barnes PJ Theophylline restores histone deacetylase activity and steroid responses in COPD macrophages. J Exp Med 200: 689-695, 2004

49. Zhang Y, He Z, Sun X, Li Z, Zhao L, Mao C, Huang D, Zhang J and Zhong X: Erythromycin restores oxidative stress-induced corticosteroid responsiveness of human THP-1 cells by up-regulating the expression of histone deacetylase 2. Xi Bao Yu Fen Z Mian Yi Xue Za Zhi 30: 384-387, 2014 (In Chinese).

50. Cui Y, Luo L, Li C, Chen P and Chen Y: Long-term macrolide treatment for the prevention of acute exacerbations in COPD: A systematic review and meta-analysis. Int J Chron Obstruct Pulmon Dis 13: 3813-3829, 2018.

51. Miao L, Gao Z, Huang F, Huang S, Zhang R, Ma D, Wu Q, Li F, Chen $\mathrm{H}$ and Wang J: Erythromycin enhances the anti-inflammatory activity of budesonide in COPD rat model. Int J Clin Exp Med 8: 22217-22226, 2015.

52. Ellis S, Lin EJ and Tartar D: Immunology of wound healing. Curr Dermatol Rep 7: 350-358, 2018.

53. Arellano-Orden E, Serrano C, Montes-Worboys A, Sánchez-López V, Laborda A, Lostalé F, Lahuerta C, Rodríguez-Panadero F and de Gregorio MA: Stent-induced tracheal stenosis can be predicted by IL- 8 expression in rabbits. Eur J Clin Invest 47: 84-92, 2017.

54. Song Y, Yu P, Lu JJ, Lu HZ, Zhu L, Yu ZH, Chen HZ and Cui YY: A mucoactive drug carbocisteine ameliorates steroid resistance in rat COPD model. Pulm Pharmacol Ther 39: 38-47, 2016.

55. Hannigan M, Zhan L, Ai Y and Huang CK: The role of p38 MAP kinase in TGF-beta1-induced signal transduction in human neutrophils. Biochem Biophys Res Commun 246: 55-58, 1998.
56. Postlethwaite AE, Keski-Oja J, Moses HL and Kang AH: Stimulation of the chemotactic migration of human fibroblasts by transforming growth factor beta. J Exp Med 165: 251-256, 1987.

57. Perng DW, Wu YC, Chang KT, Wu MT, Chiou YC, Su KC, Perng RP and Lee YC: Leukotriene C4 induces TGF-betal production in airway epithelium via p38 kinase pathway. Am J Respir Cell Mol Biol 34: 101-107, 2006.

58. Ferrara N: Vascular endothelial growth factor and the regulation of angiogenesis. Recent Prog Horm Res 55: 15-35, 35-36, 2000.

59. Leung DW, Cachianes G, Kuang WJ, Goeddel DV and Ferrara N: Vascular endothelial growth factor is a secreted angiogenic mitogen. Science 246: 1306-1309, 1989.

60. Zhang J, Li Q, Bai C, Han Y and Huang Y: Inhalation of TGF-betal antibody: A new method to inhibit the airway stenosis induced by the endobronchial tuberculosis. Med Hypotheses 73: 1065-1066, 2009

61. Lee YC, Hung MH, Liu LY, Chang KT, Chou TY, Wang YC, Wu YC, Lai CL, Tsai CC, Su KC and Perng DW: The roles of transforming growth factor-beta(1) and vascular endothelial growth factor in the tracheal granulation formation. Pulm Pharmacol Ther 24: 23-31, 2011.

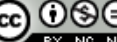

This work is licensed under a Creative Commons
Attribution-NonCommercial-NoDerivatives 4.0 International (CC BY-NC-ND 4.0) License. 